6

\section{OPEN ACCESS}

${ }^{1}$ Boston EyePain Foundation, Chestnut Hill, Massachusetts, USA

${ }^{2}$ Center for Pain and the Brain, Boston Children's, MGH and McLean Hospitals, Harvard Medical School, Children's Medical Center, Boston, Massachusetts, USA

\section{Correspondence to} Dr Perry Rosenthal, Boston EyePain Foundation, 629 Hammond St. Ste.205E, Chestnut Hill, MA 02467, USA;

prosenthal@bostoneyepain.org

Received 15 October 2014 Revised 6 February 2015 Accepted 28 February 2015 Published Online First 5 May 2015
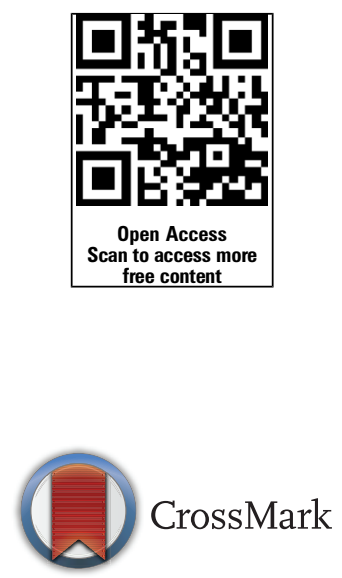

To cite: Rosenthal $P$, Borsook D. Br J Ophthalmol 2016;100:128-134.

\title{
Ocular neuropathic pain
}

\author{
Perry Rosenthal, ${ }_{1}^{1}$ David Borsook ${ }^{2}$
}

\section{ABSTRACT}

As the biological alarm of impending or actual tissue damage, pain is essential for our survival. However, when it is initiated and/or sustained by dysfunctional elements in the nociceptive system, it is itself a disease known as neuropathic pain. While the critical nociceptive system provides a number of protective functions, it is unique in its central role of monitoring, preserving and restoring the optical tear film in the face of evaporative attrition without which our vision would be nonfunctional. Meeting this existential need resulted in the evolution of the highly complex, powerful and sensitive dry eye alarm system integrated in the peripheral and central trigeminal sensory network. The clinical consequences of corneal damage to these nociceptive pathways are determined by the type and location of its pathological elements and can range from the spectrum known as dry eye disease to the centalised oculofacial neuropathic pain syndrome characterised by a striking disparity between the high intensity of symptoms and paucity of external signs. These changes parallel those observed in somatic neuropathic pain. When seen through the neuroscience lens, diseases responsible for inadequately explained chronic eye pain (including those described as dry eye) can take on new meanings that may clarify long-standing enigmas and point to new approaches for developing preventive, symptomatic and disease-modifying interventions for these currently refractory disorders.

\section{INTRODUCTION}

Our corneal pain system is unique in its principal role of protecting, sustaining and restoring the optical tear layer without which our vision would be non-functional. To meet this existential need, the human dry eye alarm evolved into the most powerful, sensitive and complex pain system in the body, incorporating sensors that are unusually vulnerable to being exposed to the noxious environment. Like other damaged nociceptive systems, it is capable of failing and becoming a disease in its own right known as neuropathic pain. ${ }^{1}$ Indeed, the features of corneal nerves associated with pain parallel the well-defined properties of somatic neuropathic pain defined as ongoing pain as a direct consequence of a lesion or disease affecting the somatosensory system. Nevertheless, a recent PubMed search (http://www.ncbi.nlm.nih.gov/ pubmed) using key words cornea, neuropathic, pain came up empty except for references to herpes zoster. Here we provide an overview of disorders of the corneal pain system in the context of neuropathic pain as it relates to dry eye disease (DED) and other disorders affecting the corneal sensory system characterised by otherwise inadequately explained chronic pain.

\section{WHAT IS DRY EYE DISEASE?}

Although the definition of DED is ambiguous and often contradictory, its most consistent clinical feature is chronic dry eye-like pain (DELP).

\section{Dry eye symptoms}

DELP is a unique type of corneal pain that we associate with excessive tear film evaporation. Unlike pain experienced elsewhere in the body, that of dry eye is characterised by its sensitivity to environmental evaporative factors in being exacerbated by circumstances that promote tear film evaporation and are mitigated by those that suppress it. Thus, in addition to mechanisms commonly associated with neuropathic pain, specialised corneal nociceptors tuned to tear film evaporation have been shown to play a central role in generating chronic sensations of dry eye (see below). Moreover, we believe that it is appropriate to distinguish them from symptoms caused by the mechanical consequences of dry ocular surfaces such as scratchy or foreign body-like and others such as burning when they are not highly sensitive to tear evaporation. The reasons for our arbitrary decision to highlight symptoms associated with evaporation of the pre-corneal tear layer will become apparent below.

Although patients with chronic corneal evaporative symptoms as a group have lower tear metrics than those who are asymptomatic, ${ }^{2}$ the weak relationship between DELP and tear metrics ${ }^{34}$ questions the assumption of a direct causal relationship. Moreover, the validity of highlighting Meibomian gland dysfunction (MGD) to explain the disparity between signs and symptoms has been challenged by the recent report of its similarly weak relationship with dry eye symptoms. ${ }^{5}$ On the other hand, dry eye symptoms are keyed to the sensitivity of specialised evaporation-sensitive (cold) receptors located in afferent corneal nerve terminals ${ }^{6}$ known as TRPM8 channels that in murine models are responsive to cooling ${ }^{7}$ and the presence of hyperosmotic tears, ${ }^{8}$ and others described as innocuous cold afferents. ${ }^{9}$ It should be noted that different results have been obtained on the effects of hyperosmolality ${ }^{6}{ }^{8}$ that may reflect differences in the experimental set-up or others that can alter the responsivity of the coldsensitive afferents (including genetic background). Hypertonicity and increased rate of cooling at the corneal surface that occurs with evaporative thinning of the overlying tear film drive the nociceptive activity that sustains basal tear secretion..$^{9-11}$ When thinning of the tear layer exceeds certain programmed thresholds, the escalating activity of these nerves triggers the release of fresh tears that normally restores the tear film thickness, thereby silencing the dry eye alarm. However, if the tearing response is inadequate, the escalating alarm activity breaches conscious levels and triggers the maximum 
release of available tears. Notably, damaged nociceptors in other body parts usually demonstrate enhanced activity to cold typical of many neuropathic conditions. ${ }^{12}$

\section{Physiological corneal sensitisation and evaporative hyperalgesia}

Hyperalgesia is the pain state characterised by increased sensitivity and responsiveness of nociceptors to their noxious stimuli. Proinflammatory cytokines released during inflammation are classic nociceptor sensitizers ${ }^{13}{ }^{14}$ presumably for those of corneas as well. As the major corneal noxious stimulus, tear evaporation also appears to be the dominant type of corneal hyperalgesia for which we propose the neogilistic term corneal evaporative hyperalgesia (CEH). $\mathrm{CEH}$ is unlikely to be clinically problematic when there is no underlying inflammation or nerve damage. It is logical to assume that the sensitivities of the cold and osmotic corneal nerve receptors increase with the greater concentrations of and (possibly) increased sensitivity of nociceptors to proinflammatory cytokines as has been demonstrated in non-ocular tissues. ${ }^{15}$ In such cases the thickness of the thermal insulating and diluting tear film required to avoid triggering the 'sensitised' dry eye alarm must also be greater. This suggests that when the increased thickness of the tear film required to maintain the silence of the sensitised dry eye alarm becomes unsustainable, DELP can be experienced even in the presence of normally adequate tears metrics assuming that the neuralmediated tear secretory function of the lacrimal glands ${ }^{16}$ remains adequate.

\section{The scleral lens: a diagnostic tool for CEH}

The unique capability of scleral lenses to instantly and completely block corneal surface evaporation on their insertion points to symptoms they suppress as representing CEH. Moreover, by maintaining an optimal hydrating environment and protecting the external corneal surface from the friction of blinking (thereby avoiding the activation of sensitised lowthreshold C-mechanoreceptors), corneal symptoms experienced while these devices are worn could be expected to represent spontaneous pain (the exception being chemical hyperalgesia as these devices are permeable to fumes).

\section{(NEUROPATHIC) DISORDERS OF THE PERIPHERAL DRY DYE ALARM}

Although the traditional model of DED assumes that the dry eye alarm is a passive participant, cold receptors elsewhere in the body have been shown to be capable of becoming autonomous drivers of neuropathic nociceptor hyperactivity. ${ }^{17}$ We suggest that the cascade involved in the expression of evaporative corneal hyperalgesia can be similarly driven by dysfunctional nociceptor elements.

\section{Tear deficiency and chronic DELP}

It has been assumed that otherwise unexplained chronic DELP is caused by insufficient tears because they are identical to those associated with dry eyes and some of these eyes exhibit vital staining of their exposed surfaces. However, these symptoms can also be experienced despite the presence of adequate tears as reported a study of 52 eyes in which tear volumes did not differ significantly from preoperative values over a period of 6 months following laser-assisted in situ keratomileusis (LASIK). ${ }^{18}$ Moreover, it is possible that the exposed ocular surfaces may be more vulnerable to desiccating stress by neurotrophic effects of corneal nerve damage from the procedure. Nevertheless, it is not surprising that studies have found reduced tears in these eyes ${ }^{19}$ presumably consequent to the disruption of afferent neural pathways of the lacrimal functional unit.

In our opinion, the role of Meibomian glands in the pathoetiology of chronic DELP is also debatable. Although MGD is associated with increased tear evaporation, ${ }^{20}$ the relationship between chronic DELP and MGD is weak, ${ }^{5}$ suggesting that the function of Meibomian glands may not be a significant factor as an initiating cause in chronic DELP. On the other hand, interventions that improve Meibomian gland function can temporarily reduce these symptoms. ${ }^{21}$ How can these findings be reconciled? We suggest that the missing part of the equation may be due to the effects of corneal sensitisation. While nonsensitised corneas appear to be agnostic to MGD, that may not be true of sensitised corneas since they could be expected to be more symptomatically responsive to changes in tear film evaporation; both positive and negative. Increased concentrations of proinflammatory cytokines in the tears of these patients ${ }^{22} 23$ suggest the presence of corneal sensitisation.

\section{When is cornea-based chronic DELP pain neuropathic?}

Hyperosmotic tears, a marker of tear deficiency, ${ }^{24-26}$ have been shown to induce inflammation in human corneal epithelial cells, ${ }^{27}$ increase the local ability to concentrate human neutrophils $^{28}$ and enhance the sensitivity of cold transducers in corneal nociceptors. ${ }^{29}$ Nevertheless, the tear osmolarity distribution curves of symptomatic patients include some that are not hyperosmolar. This should be considered in the context of a progressive or chronic process whereby the nerves are either sensitised by inflammatory molecules damage. Many diseases affect nerves involved in chronic pain throughout the body (eg, diabetes, fibromyalgia) or from local damage (eg, LASIK). The above observation is consistent with anecdotal observations of the presence in some patients with chronic DELP of tears that are too abundant to be explained by hyperevaporating tears (PR, personal observations). Should future studies confirm the presence of elevated levels of proinflammatory cytokines in nonhypertonic tears of patients reporting chronic DELP, their possible source could be neuroinflammation caused by prolonged periods of corneal nociceptor hyperactivity (peripheral sensitisation) as has been shown in other tissues (see reviews ${ }^{30}{ }^{31}$ ). Moreover, the typical paucity of external signs of inflammation in these eyes has also been noted in other types of peripheral neuropathies characterised by neuroinflammation and peripheral sensitisation. ${ }^{32}$ Being more sensitive to noxious stimuli, neuropathically sensitised corneas might also be expected to be more responsive to localised areas of hyperosmolarity occurring in areas of tear film break-up ${ }^{33}$ that would be asymptomatic in non-sensitised corneas.

\section{Age-related dry eye disease}

These corneas are characterised by negative symptoms of reduced tactile sensitivity related to lower corneal nerve densities $^{34}$ and positive symptoms of evaporative hyperalgesia. The juxtaposition of opposite sensory features was also reported in the skin of healthy elderly subjects in whom it was found that although the overall activity of the C-fibres was reduced by their attrition, that of the surviving nerves was increased, ${ }^{35}$ indicating ongoing hyperactivity. If similar age-related changes occur in the corneal nerves, it could explain the presence of chronic dry eye symptoms in this cohort considering the far greater density of corneal nociceptors compared with skin. Moreover, the effect of aging on enhancing central pain-modulating processing ${ }^{36}$ may also be a factor in amplifying the intensity of $\mathrm{CEH}$. 
Unexplained, chronic corneal evaporative hyperalgesia is consistent with neuropathic pain.

\section{Neuropathic pain following corneal nerve injury}

Pain following non-ocular surgery is typically generated by inherently hypersensitive ectopic pacemakers located in regenerating nociceptor fibre terminals and dorsal root ganglia ${ }^{37}$ in which its phenotype has transitioned from transmission to regeneration. ${ }^{38}$ Healing is accompanied by their return to the transmitting phenotype accompanied by pruning of redundant regenerating nerve fibres, microneuromas and their ectopic pain signal generators and the termination of ectopic pacemaker expression in their somata. Nevertheless, unexplained chronic pain following otherwise uneventful non-ocular surgical procedures that persists long after complete healing has taken place indicates the presence of neuropathic pain. ${ }^{39}$

DELP is the dominant symptom experienced by patients following the extensive corneal axotomies performed during laser keratorefractive surgery. Although they typically resolve within several months, symptoms can persist as long as a year ${ }^{40}$ and sometimes longer in which case their levels typically reach higher levels of intensity ${ }^{41}$ and in our experience can be permanent.

\section{NEUROPATHIC EYE PAIN AND THE BRAIN}

Peripheral injuries trigger complex changes in the central nociceptive system leading to central sensitisation that enhances the sensitivity and responsiveness of the brain regions involved in sensory processing through neuroplastic processes. ${ }^{42}{ }^{43}$ In some cases, these physiological responses progress to neuropathic centralised pain.

\section{Physiological central sensitisation}

In contrast to all other sensory systems that adapt to sustained applications of constant stimuli, that of pain is amplified both peripherally (peripheral sensitisation) and centrally (central sensitisation). Central sensitisation is the physiological phenomenon that describes the augmentation of incoming trains of peripheral afferent pain signals in the dorsal horn ${ }^{44}$ and its equivalent in the trigeminal brainstem ${ }^{45}$ by activity-driven feed-back and feedforward mechanisms. The result is sustained elevated excitability levels in relevant synapses through adaptive molecular and cellular neuroplastic changes. ${ }^{46}$ Its upper limits are controlled by responsive descending inhibitory pain signals ${ }^{47}$ that dominate the background of the central nociceptive system and serve to extinguish central sensitisation when afferent pain signals cease (see review). ${ }^{47}$

The dynamic responsiveness of central sensitisation to increased peripheral pain activity triggered by nerve injuries and inflammation is a function of the remarkable neuroplasticity of the somatosensory nervous system. ${ }^{48}$ Nevertheless, these changes can become entrenched and counterproductive; a phenomenon described as maladaptive plasticity. ${ }^{42}$ Inappropriately sustained synaptic membrane hyperexcitability in the central nociceptive pathways can be caused by an imbalance between excitation and inhibition promoted by a compromised inhibitory system (disinhibition). ${ }^{49} 50$ The consequent facilitation of central pain signals amplifies and distorts incoming pain signals such as those generated by tear film evaporation (central CEH), that can result in painful responses to normally non-noxious stimuli (allodynia), and spontaneous discharges in the central nociceptive pathways (spontaneous pain). ${ }^{51}$
Oculofacial pain

Pathologically entrenched synaptic hyperexcitability in the central pain pathways results in sustained amplification and distortion of incoming pain signals (central hyperalgesia) and/or spontaneous pain long after the triggering noxious insult has resolved. Involvement of the eyes in this pain syndrome points to malfunctions in the trigeminal brainstem, which is the target of ascending corneal nociceptive projections in murine models. ${ }^{52}$

Our use of the term oculofacial pain (OFP) describes neuropathic pain generated and/or sustained in the central trigeminal circuitry and projected to its receptive field including (and sometimes limited to) that of its ophthalmic division. Because centralised pain is expressed through neurological mechanisms remote from its origins (see review ${ }^{53}$ ), its apparent peripheral trigger is an illusion responsible for the striking disparity between symptoms and signs. Moreover, as the centre for processing corneal hyperalgesia, ${ }^{54}$ maintaining corneal surface wetness homeostasis ${ }^{55}$ and monitoring aversive light intensity, ${ }^{56}$ dysfunctional synaptic excitability levels in the trigeminal brainstem can, in addition to generating projected spontaneous pain, impair tear production and increase the pain of photosensitivity (neuropathic photophobia)—see below.

Clinical observations of over 200 patients (PR) of presumed centralised OFP have provided further insights into symptoms that these patients can experience. We identified the components of OFP as spontaneous when they were experienced during lid closure in a dark room or during scleral lens wear. Patient descriptors of spontaneous pain included burning hot, sharp, cutting, needle-like, foreign body, pins and needles and pounding, pressure/aching. It was often described as radiating to and from the eyes and/or orbits, ears and receptive fields of the maxillary and mandibular divisions of the trigeminal nerve. Comorbid headaches were common and most often characterised as new and less commonly as an exacerbation of longstanding ones. Tactile allodynia of skin innervated by the ophthalmic branch of the trigeminal nerve was reported by a few patients. Notably, hypersensitivity to sound (hyperacusis), although rarely volunteered, was common. Tinnitus, vertigo and/or alterations in smell and taste were also described. On the other hand, in some patients' symptoms included and were sometimes dominated by those of $\mathrm{CEH}$ in which case they appeared to differ from those generated solely in the cornea by their greater intensity and more common association with chemical and occasionally cold corneal hyperalgesia. Symptoms were limited to those of neuropathic photophobia in a few patients (see below).

\section{Neuropathic photophobia}

Allodynia was most often reported as otherwise unexplained painful photophobia typically characterised by increased sensitivity to lights of computer screens and fluorescent and metal halide fixtures (characterised by high-frequency flicker) and less to incandescent lights (PR, personal observations). The association of photophobia with centralised trigeminal pain has been previously reported and in the absence of a visible peripheral cause we consider it being a neuropathic symptom and describe it as photoallodynia. ${ }^{57} 58$ It also appeared to differ from peripherally triggered photoallodynia in the failure of cycloplegia to mitigate symptoms (PR, personal observations) consistent with the findings in a murine model. ${ }^{59}$ Based on these observations, we speculate that, while we believe that neuropathic photophobia is associated with the activation of the corneal-trigeminal pathways similar 
to that associated with corneal trauma, ${ }^{60}$ it differs in being sustained by neuropathic disorders involving relevant pathways in the brainstem. ${ }^{59}$ These symptoms appear to share certain properties with those described by migrainous patients in whom exposure to light exacerbates their headache ${ }^{61}$ and who have been reported to be more sensitive to highfrequency flickering light. ${ }^{62}$

\section{Centralised mechano-hyperalgesia associated with OFP}

Many patients in our cohort of OFP were inexplicably intolerant to wearing scleral lenses. Although this response was typically experienced during the initial fitting process, it also appeared after the devices had been worn comfortably; sometimes for several years (personal observations). Some of these patients also experienced an unusually painful reaction to the application of Schirmer's strips (PR, personal observations) consistent with secondary hyperalgesia associated with centralised neuropathic pain. ${ }^{63}$

\section{OFP following laser keratorefractive surgery}

Since neuropathic pain is known to follow surgical incisions of the skin ${ }^{64}$ which is far less densely innervated than the cornea, it is not surprising that the massive corneal axotomies performed during laser keratorefractive procedures can trigger this disease in vulnerable patients. We identified a cohort $(\mathrm{n}=21)$ who developed severe neuropathic eye pain consistent with its centralisation following keratorefractive surgery. The duration of the pain at the initial consultation ranged from 2 to 15 years (unpublished data). Eighty-one per cent of these patients reported significant neuropathic photophobia. An unexpected finding was that the onset of the centralised pain following the resolution of postoperative pain was often delayed for periods ranging from days to years. If validated, these findings suggest that some currently asymptomatic post-keratorefractive surgery patients are at risk for developing this devastating complication. Although not described as such, the neuropathic centralised pain state following LASIK has been previously described ${ }^{41}$ as has the delayed onset of chronic pain following surgery of other body parts. ${ }^{65}$

\section{Responses to topical corneal anaesthesia: a diagnostic test for centralised corneal pain}

Most patients with OFP reported incomplete suppression of their eye symptoms during total topical corneal anaesthesia. Two examples are provided below.

The experience of a 28-year-old physician provides an informative example. Her chronic, bilateral OFP of 4 years was triggered by a minor chemical injury reported to have rapidly healed and was described as consisting of two distinct components: symptoms of $\mathrm{CEH}$ and spontaneous sharp, needle-like sensations that she localised to her eyes and face. Topical corneal anaesthesia suppressed symptoms of CEH as expected but failed to do so for those of spontaneous needle-like pain. That response, the normal appearance of her corneal nerves and absence of inflammatory cells in laser scanning confocal microscope images of her corneas suggested that the source of her spontaneous eye pain was located central to the trigeminal ganglion.

In contrast, a 51-year-old otherwise healthy man developed OFP 5 years after LASIK at which time confocal microscope images revealed striking morphological signs of active corneal axon degeneration/regeneration and inflammation. He reported total suppression of his spontaneous and hyperalgesic oculofacial symptoms including dental pain during corneal anaesthesia. This patient's symptoms suggested possible involvement of corneal polymodal nociceptive afferents ${ }^{66}{ }^{67}$ and a key role of corneal nociceptor activity in sustaining the central neuropathic process. Differences in the symptom patterns of these two patients reflect the diversity of mechanisms involved in the pathogenesis of peripherally triggered centralised pain.

\section{OFP AND COMORBID DISORDERS Fibromyalgia}

Fibromyalgia is now believed to be a brain disorder characterised by aberrant central pain facilitation and a state of hyperalgesia ${ }^{68}$ believed to be sustained by impaired descending inhibition. ${ }^{69}$ In our experience, the OFP syndrome, whether or not preceded by a noxious insult in the trigeminal sensory receptive field, was often associated with symptoms of fibromyalgia and/or associated comorbidities such as irritable bowel syndrome ${ }^{70}$ chronic fatigue, temporomandibular disorders, ${ }^{71}$ multichemical sensitivities, ${ }^{72}$ pelvic pain and urological disorders, ${ }^{73}$ carpal tunnel syndrome ${ }^{74}$ and sleep disorders ${ }^{75}$ all of which have been linked to neuropathic central sensitisation. ${ }^{76}$ In our opinion, this suggests that OFP may also reflect a hypersensitive pain state in some patients. ${ }^{77}$

\section{Peripheral neuropathies}

Some patients in our cohort were diagnosed with small fibre neuropathy that could serve as driver of centralised pain and that has also been reported in patients with fibromyalgia. ${ }^{78}$ The disparity between dry eye symptoms and tear metrics in this disease has been previously reported. ${ }^{79}$

\section{OFP and blepharospasm}

The association of blepharospasm and OFP was reported in a case series of five patients (four women and one male).$^{80}$ In vivo confocal microscopy revealed strikingly abnormal corneal nerve morphology in all, of whom one had previously undergone LASIK. All these patients reported spontaneous pain, chronic dry eye-like symptoms and photophobia in the absence of vital dye staining of their exposed corneal surface. Dysfunctional activity in the basal ganglia-thalamocortical circuitry has been observed in patients with pain and cervical dystonia. ${ }^{81}$

\section{SJOGREN'S SYNDROME}

While studies have reported lower tear volumes in patients with Sjogren's syndrome (SS), ${ }^{82}$ this has not been a uniform finding. ${ }^{83}$ Moreover, signs and symptoms of dry eye were not found to be related to the level of autoimmune activity in this disease. ${ }^{84}$ The weak correlation between symptoms and signs of dry eye in these patients was also consistent with our informal observations that although all patients who were referred to us with this diagnosis suffered from disabling chronic dry eye symptoms and most from desiccating DED, some had normal tear metrics and ocular surfaces. On the other hand, a few patients in the latter group did develop severe dry eyes over time (PR, personal observations), suggesting that the discordance between symptoms and signs may be more significant in the early phases of the disease. If validated, these observations could be interpreted as supporting corneal sensitisation as a cause of chronic DELP in SS that can occur prior to the onset of tear deficiency. This hypothesis could be further tested by measuring both the levels of proinflammatory cytokines in the tears of these patients (which have been shown to be elevated in past studies ${ }^{85}$ ) and their osmolarities. The finding of elevated levels of proinflammatory cytokines in non-hyperosmolar tears would point to another source of inflammation such as neuroinflammation caused by the hyperactivity of dysfunctional corneal nociceptors. This would also be consistent with the increased 
incidence of peripheral neuropathies in this disease. ${ }^{86}$ Moreover, these corneas could also be expected to be hypersensitive to tear evaporation accelerators such as MGD, a known complication of SS. ${ }^{87} 88$

The foregoing comments are consistent with the increased prevalence of signs and symptoms of central nervous system disorders in associated syndromes. ${ }^{89}$

\section{COGNITIVE AND AFFECTIVE COMPLICATIONS OF NEUROPATHIC OFP}

Chronic pain is associated with numerous brain-related changes that can impair memory, alter reward processing and compromise cognitive functions. ${ }^{90}$ Many of our patients with long-standing OFP also reported a significant deterioration of their cognitive and executive functions and working memory consistent with reports of fibromyalgia patients. ${ }^{91}$ In this context, the management of these patients should take into account the bidirectional effects of depression and chronic pain. ${ }^{92} 93$ Moreover, it has been shown that patients diagnosed as having DED are burdened by low self-assessment scores of their health and well-being. ${ }^{94}$

\section{DOES MITOCHONDRIAL DYSFUNCTION \\ PLAY A ROLE IN OFP?}

The diversity of systemic diseases associated with centralised corneal pain suggests that they may share underlying pathogenetic vulnerabilities. Reports of mitochondrial dysfunction in patients with $\mathrm{SS}^{95}$ and fibromyalgia ${ }^{96}$ and in those with chronic neuropathic pain ${ }^{97}$ suggest a possible link, whether one of association or causation.

\section{CONCLUSIONS}

If validated, our suggestion that chronic dry eye-like symptoms are promoted and sustained by $\mathrm{CEH}$ that can be neuropathic in some patients expands our conceptual horizons of these enigmatic diseases in which the role of tear deficiency is relegated to the level of an associated complication from its current assigned role as the underlying cause. We also suggest that the typical disparity between symptoms and signs in the so-called DED are consequences of the varying relationship between levels of corneal sensitisation and compromised tear production each of which in our opinion is a complication of the disease that can evolve along different pathways and timelines.

Separately we describe a previously overlooked oculofacial trigeminal pain syndrome similarly characterised by a striking discordance between the high-intensity levels of its symptoms and paucity of external signs. As the location of the first synapse of primary corneal afferents that transmit noxious corneal sensations, the homeostatic corneal surface wetness control centre and the centre for monitoring the averseness of ambient light levels the trigeminal brainstem would appear to be the location of the neuropathic disease capable of causing trigeminalprojected pain and otherwise unexplained photophobia. The various clinical expressions of this sensory disease reflect the complexity of overlapping networks of interactive pathogenetic cascades adding to the bewilderment of clinicians many of whom take refuge in the belief that these symptoms are imaginary or fabricated.

If the neuropathic paradigm is validated, may open new avenues for the development of targeted treatments for this spectrum of enigmatic diseases.

Correction notice This article has been corrected since it was published Online First. Perry Rosenthal is listed at affiliation 1 only. The provenance and peer review statement has been corrected.
Acknowledgements The authors are grateful to Todd Margolis, M.D., PhD, Carlos Belmonte Martinez, PhD and Kazuo Tsubota, M.D., PhD for their support of this review and helpful suggestions.

Contributors Defining the concept: PR. Drafting and editing the manuscript: PR, DB.

Competing interests None.

Provenance and peer review Not commissioned; externally peer reviewed.

Open Access This is an Open Access article distributed in accordance with the Creative Commons Attribution Non Commercial (CC BY-NC 4.0) license, which permits others to distribute, remix, adapt, build upon this work non-commercially, and license their derivative works on different terms, provided the original work is properly cited and the use is non-commercial. See: http://creativecommons.org/ licenses/by-nc/4.0/

\section{REFERENCES}

1 Treede RD, Jensen TS, Campbell JN, et al. Neuropathic pain: redefinition and a grading system for clinical and research purposes. Neurology 2008;70:1630-5.

2 Mainstone JC, Bruce AS, Golding TR. Tear meniscus measurement in the diagnosis of dry eye. Curr Eye Res 1996;15:653-61.

3 Nichols KK, Nichols JJ, Mitchell GL. The lack of association between signs and symptoms in patients with dry eye disease. Cornea 2004;23:762-70.

4 Schein OL, Tielsch JM, Munos B, et al. Relation between signs and symptoms of dry eye in the elderly: a population-based perspective. Ophthalmol 1997;104:1395-401.

5 Galor A, Feuer W, Lee DJ, et al. Ocular surface parameters in older male veterans. Invest Ophthalmol Vis Sci 2013;54:1426-33.

6 Hirata H, Oshinsky ML. Ocular dryness excites two classes of corneal afferent neurons implicated in basal tearing in rats: involvement of transient receptor potential channels. J Neurophysio/ 2012;107:1199-209.

7 Parra A, Madrid R, Echevarria D, et al. Ocular surface wetness is regulated by TRPM8-dependent cold thermoreceptors of the cornea. Nat Med 2010;16:1396-9.

8 Parra A, Gonzalez-Gonzalez 0, Gallar J, et al. Tear fluid hyperosmolality increases nerve impulse activity of cold thermoreceptor endings of the cornea. Pain 2014; 155:1481-91.

9 Hirata H, Meng ID. Cold-sensitive corneal afferents respond to a variety of ocular stimuli central to tear production: implications for dry eye disease. Invest Ophthalmol Vis Sci 2010;51:3969-76.

10 Belmonte C, Gallar J. Cold thermoreceptors, unexpected players in tear production and ocular dryness sensations. Invest Ophthalmol Vis Sci 2011;52:3888-92.

11 Robbins A, Kurose M, Winterson BJ, et al. Menthol activation of corneal cool cells induces TRPM8-mediated lacrimation but not nociceptive responses in rodents. Invest Ophthalmol Vis Sci 2012;53:7034-42.

12 Andersen $\mathrm{HH}$, Olsen RV, Moller HG, et al. A review of topical high-concentration L-menthol as a translational model of cold allodynia and hyperalgesia. Eur J Pain 2014; 18:315-25.

13 Sommer C, Kress M. Recent findings on how proinflammatory cytokines cause pain: peripheral mechanisms in inflammatory and neuropathic hyperalgesia. Neurosci Lett 2004:361:184-7.

14 Watkins LR, Wiertelak EP, Goehler LE, et al. Characterization of cytokine-induced hyperalgesia. Brain Res 1994;654:15-26.

15 Reichling DB, Levine JD. Critical role of nociceptor plasticity in chronic pain. Trends Neurosci 2009;32:611-18.

16 Dartt DA. Neural regulation of lacrimal gland secretory processes: relevance in dry eye diseases. Prog Retin Eye Res 2009;28:155-77.

17 Serra J, Solà R, Quiles C, et al. C-nociceptors sensitized to cold in a patient with small-fiber neuropathy and cold allodynia. Pain 2009;147:46-53.

18 Patel S, Alio JL, Artola A, et al. Tear volume and stability after LASIK. J Refract Surg 2007;23:290-8

19 Nejima R, Miyata K, Tanabe T, et al. Corneal barrier function, tear film stability, and corneal sensation after photorefractive keratectomy and laser in situ keratomileusis. Am J Ophthalmol 2005;139:64-71.

20 Mathers WD. Ocular evaporation in meibomian gland dysfunction and dry eye. Ophthalmology 1993;100:347-51.

21 Lane SS, DuBiner HB, Epstein RJ, et al. A new system, the LipiFlow, for the treatment of meibomian gland dysfunction. Cornea 2012;31:396-404.

22 Enriquez-de-Salamanca A, Castellanos E, Stern ME, et al. Tear cytokine and chemokine analysis and clinical correlations in evaporative-type dry eye disease. Mol Vis 2010;16:862-73.

23 Lam H, Bleiden L, de Paiva CS, et al. Tear cytokine profiles in dysfunctional tear syndrome. Am J Ophthalmol 2009;147:198-205.e191.

24 Tomlinson A, Khanal S, Ramaesh K, et al. Tear film osmolarity: determination of a referent for dry eye diagnosis. Invest Ophthalmol Vis Sci 2006;47:4309-15.

25 Lemp MA, Bron AJ, Baudouin C, et al. Tear osmolarity in the diagnosis and management of dry eye disease. Am J Ophthalmol 2011;151:792-8.e791. 
26 Gilbard JP, Farris RL, Santamaria J 2nd. Osmolarity of tear microvolumes in keratoconjunctivitis sicca. Arch Ophthalmol 1978;96:677-81.

27 Li DQ, Chen Z, Song XJ, et al. Stimulation of matrix metalloproteinases by hyperosmolarity via a JNK pathway in human corneal epithelial cells. Invest Ophthalmol Vis Sci 2004;45:4302-11.

28 Tibrewal S, Ivanir Y, Sarkar J, et al. Hyperosmolar stress induces neutrophil extracellular trap formation: implications for dry eye disease. Invest Ophthalmol Vis Sci 2014:55:7961-9.

29 Hirata H, Rosenblatt Ml. Hyperosmolar tears enhance cooling sensitivity of the corneal nerves in rats: possible neural basis for cold-induced dry eye pain. Invest Ophthalmol Vis Sci 2014;55:5821-33.

30 Moalem G, Tracey DJ. Immune and inflammatory mechanisms in neuropathic pain. Brain Res Rev 2006;51:240-64.

31 Bhave G, Gereau RWt. Posttranslational mechanisms of peripheral sensitization. J Neurobiol 2004:61:88-106.

32 Herder C, Lankisch M, Ziegler $\mathrm{D}$, et al. Subclinical inflammation and diabetic polyneuropathy: MONICA/KORA Survey F3 (Augsburg, Germany). Diabetes Care 2009:32:680-2.

33 Liu $\mathrm{H}$, Begley $\mathrm{C}$, Chen $\mathrm{M}$, et al. A link between tear instability and hyperosmolarity in dry eye. Invest Ophthalmol Vis Sci 2009;50:3671-9.

34 Benitez del Castillo JM, Acosta MC, Wassfi MA, et al. Relation between corneal innervation with confocal microscopy and corneal sensitivity with noncontact esthesiometry in patients with dry eye. Invest Ophthalmol Vis Sci 2007:48:173-81.

35 Namer B, Barta B, Orstavik K, et al. Microneurographic assessment of C-fibre function in aged healthy subjects. J Physiol 2009;587(Pt 2):419-28.

36 Cole LJ, Farrell MJ, Gibson SJ, et al. Age-related differences in pain sensitivity and regional brain activity evoked by noxious pressure. Neurobiol Aging 2010:31:494-503.

37 Wolf G, Gabay E, Tal M, et al. Genetic impairment of interleukin-1 signaling attenuates neuropathic pain, autotomy, and spontaneous ectopic neuronal activity, following nerve injury in mice. Pain 2006;120:315-24.

38 Zhou XF, Chie ET, Deng YS, et al. Injured primary sensory neurons switch phenotype for brain-derived neurotrophic factor in the rat. Neuroscience 1999;92:841-53.

39 Kehlet $\mathrm{H}$, Jensen TS, Woolf $\mathrm{CJ}$. Persistent postsurgical pain: risk factors and prevention. Lancet 2006;367:1618-25.

40 Murakami Y, Manche EE. Prospective, randomized comparison of self-reported postoperative dry eye and visual fluctuation in LASIK and photorefractive keratectomy. Ophthalmology 2012;119:2220-4.

41 Nettune GR, Pflugfelder SC. Post-LASIK tear dysfunction and dysesthesia. Ocul Surf 2010:8:135-45.

42 Latremoliere A, Woolf CJ. Central sensitization: a generator of pain hypersensitivity by central neural plasticity. J Pain 2009;10:895-926.

43 Navarro X, Vivo M, Valero-Cabre A. Neural plasticity after peripheral nerve injury and regeneration. Prog Neurobiol 2007;82:163-201.

44 Woolf $\mathrm{CJ}$. Evidence for a central component of post-injury pain hypersensitivity. Nature 1983;306:686-99.

45 Sessle BJ. Trigeminal central sensitization. Rev Analg 2005:8:85-102.

46 Ji RR, Kohno T, Moore KA, et al. Central sensitization and LTP: do pain and memory share similar mechanisms? Trends Neurosci 2003;26:696-705.

47 Lau BK, Vaughan CW. Descending modulation of pain: the GABA disinhibition hypothesis of analgesia. Curr Opin Neurobiol 2014;29C:159-64.

48 Coderre TJ, Katz J, Vaccarino AL, et al. Contribution of central neuroplasticity to pathological pain: review of clinical and experimental evidence. Pain 1993:52:259-85.

49 Wang $\mathrm{R}$, King $\mathrm{T}$, De Felice $\mathrm{M}$, et al. Descending facilitation maintains long-term spontaneous neuropathic pain. J Pain 2013;14:845-53.

50 Lefaucheur JP, Drouot X, Menard-Lefaucheur I, et al. Motor cortex rTMS restores defective intracortical inhibition in chronic neuropathic pain. Neurology 2006;67:1568-74.

51 Seifert F, Maihöfner C. Functional and structural imaging of pain-induced neuroplasticity. Curr Opin Anaesthesiol 2011;24:515-23.

52 Aicher SA, Hegarty DM, Hermes SM. Corneal pain activates a trigemino-parabrachial pathway in rats. Brain Res 2014;1550:18-26.

53 Truini A, Cruccu G. Pathophysiological mechanisms of neuropathic pain. Neurol Sci 2006:27(Suppl 2):S179-82.

54 Tashiro A, Okamoto K, Chang Z, et al. Behavioral and neurophysiological correlates of nociception in an animal model of photokeratitis. Neuroscience 2010;169:455-62.

55 Hirata H, Okamoto K, Tashiro A, et al. A novel class of neurons at the trigeminal subnucleus interpolaris/caudalis transition region monitors ocular surface fluid status and modulates tear production. I Neurosci 2004;24:4224-32.

56 Rahman M, Okamoto K, Thompson R, et al. Trigeminal pathways for hypertonic saline- and light-evoked corneal reflexes. Neuroscience 2014;277:716-23.

57 Rosenthal P, Baran I, Jacobs DS. Corneal pain without stain: is it real? Ocul Surf 2009; $7: 28-40$.

58 Rosenthal P, Borsook D. The corneal pain system. Part l: the missing piece of the dry eye puzzle. Ocul Surf 2012;10:2-14.
59 Okamoto K, Tashiro A, Chang Z, et al. Bright light activates a trigeminal nociceptive pathway. Pain 2010;149:235-42.

60 Moulton EA, Becerra L, Borsook D. An fMRI case report of photophobia: activation of the trigeminal nociceptive pathway. Pain 2009;145:358-63.

61 Boulloche N, Denuelle M, Payoux P, et al. Photophobia in migraine: an interictal PET study of cortical hyperexcitability and its modulation by pain. J Neurol Neurosurg Psychiatry 2010;81:978-84

62 Thabet M, Wilkinson F, Wilson HR, et al. The locus of flicker adaptation in the migraine visual system: a dichoptic study. Cephalalgia 2013;33:5-19.

63 Urban MO, Gebhart GF. Supraspinal contributions to hyperalgesia. Proc Natl Acad Sci USA 1999:96:7687-92.

64 Borsook D, Kussman BD, George E, et al. Surgically induced neuropathic pain: understanding the perioperative process. Ann Surg 2013:257:403-12.

65 Schott GD. Delayed onset and resolution of pain: some observations and implications. Brain2001;124(Pt 6):1067-76.

66 Acosta MC, Belmonte C, Gallar J. Sensory experiences in humans and single-unit activity in cats evoked by polymodal stimulation of the cornea. J Physiol 2001;534 (Pt. 2):511-25.

67 Pozo MA, Gallego R, Gallar J, et al. Blockade by calcium antagonists of chemical excitation and sensitization of polymodal nociceptors in the cat's cornea. J Physiol 1992:450:179-89.

68 Jorge LL, Amaro E Jr. Brain imaging in fibromyalgia. Curr Pain Headache Rep 2012;16:388-98.

69 Julien N, Goffaux P, Arsenault P, et al. Widespread pain in fibromyalgia is related to a deficit of endogenous pain inhibition. Pain 2005;114:295-302.

70 Riedl A, Schmidtmann M, Stengel A, et al. Somatic comorbidities of irritable bowel syndrome: a systematic analysis. J Psychosom Res 2008;64:573-82.

71 Rodriguez MA, Afari N, Buchwald DS; National Institute of D, Digestive, Kidney Diseases Working Group on Urological Chronic Pelvic P. Evidence for overlap between urological and nonurological unexplained clinical conditions. I Urol 2009;182:2123-31.

72 Slotkoff AT, Radulovic DA, Clauw DJ. The relationship between fibromyalgia and the multiple chemical sensitivity syndrome. Scand I Rheumatol 1997;26:364-7.

73 Nickel JC, Tripp DA, Pontari M, et al. Interstitial cystitis/painful bladder syndrome and associated medical conditions with an emphasis on irritable bowel syndrome, fibromyalgia and chronic fatigue syndrome. J Urol 2010;184:1358-63.

74 Perez-Ruiz F, Calabozo M, Alonso-Ruiz A, et al. High prevalence of undetected carpal tunnel syndrome in patients with fibromyalgia syndrome. J Rheumatol 1995:22:501-4.

75 Roizenblatt S, Neto NSR, Tufik S. Sleep disorders and fibromyalgia. Curr Pain Headache Rep 2011:15:347-57.

76 Woolf $\mathrm{CJ}$. Central sensitization: implications for the diagnosis and treatment of pain Pain 2011;152(3 Suppl):S2-15.

77 Napadow V, Harris RE. What has functional connectivity and chemical neuroimaging in fibromyalgia taught us about the mechanisms and management of 'centralized'pain? Arthritis Res Ther 2014;16:425.

78 Oaklander AL, Herzog ZD, Downs HM, et al. Objective evidence that small-fiber polyneuropathy underlies some illnesses currently labeled as fibromyalgia. Pain 2013;154:2310-16

79 Price EJ, Venables PJ. Dry eyes and mouth syndrome-a subgroup of patients presenting with sicca symptoms. Rheumatology (Oxford) 2002;41:416-22.

80 Borsook D, Rosenthal P. Chronic (neuropathic) corneal pain and blepharospasm: five case reports. Pain 2011;152:2427-31.

81 Colosimo C, Suppa A, Fabbrini G, et al. Craniocervical dystonia: clinical and pathophysiological features. Eur J Neurol 2010;17(Suppl 1):15-21.

82 Uchida $A$, Uchino $M$, Goto $E$, et al. Noninvasive interference tear meniscometry in dry eye patients with Sjogren syndrome. Am J Ophthalmol 2007;144:232-7.

83 Versura $\mathrm{P}$, Frigato $\mathrm{M}$, Cellini $\mathrm{M}$, et al. Diagnostic performance of tear function tests in Sjogren's syndrome patients. Eye 2007;21:229-37.

84 van Bijsterveld OP, Kruize AA, Bleys RL. Central nervous system mechanisms in Sjogren's syndrome. Br J Ophthalmol 2003:87:128-30.

85 Lee SY, Han SJ, Nam SM, et al. Analysis of tear cytokines and clinical correlations in Sjogren syndrome dry eye patients and non-Sjogren syndrome dry eye patients. Am J Ophthalmol 2013;156:247-53.e241.

86 Kaplan JG, Rosenberg R, Reinitz E, et al. Invited review: peripheral neuropathy in Sjogren's syndrome. Muscle Nerve 1990;13:570-9.

87 Goto E, Matsumoto Y, Kamoi M, et al. Tear evaporation rates in Sjogren syndrome and non-Sjogren dry eye patients. Am I Ophthalmol 2007;144:81-5.

88 Shimazaki J, Goto E, Ono M, et al. Meibomian gland dysfunction in patients with Sjogren syndrome. Ophthalmology 1998;105:1485-8.

89 Morreale M, Marchione P, Giacomini P, et al. Neurological involvement in primary Sjogren syndrome: a focus on central nervous system. PLOS ONE 2014;9: e84605.

90 Tracey I, Bushnell MC. How neuroimaging studies have challenged us to rethink: is chronic pain a disease? J Pain 2009;10:1113-20.

91 Glass JM. Review of cognitive dysfunction in fibromyalgia: a convergence on working memory and attentional control impairments. Rheum Dis Clin North Am 2009;35:299-311. 
92 Kroenke K, Wu J, Bair MJ, et al. Reciprocal relationship between pain and depression: a 12-month longitudinal analysis in primary care. J Pain 2011;12:964-73.

93 Galor A, Feuer W, Lee DJ, et al. Depression, post-traumatic stress disorder, and dry eye syndrome: a study utilizing the national United States Veterans Affairs administrative database. Am J Ophthalmol 2012;154:340-346.e342.

94 Mertzanis P, Abetz L, Rajagopalan K, et al. The relative burden of dry eye in patients' lives: comparisons to a U.S. normative sample. Invest Ophthalmol Vis Sci 2005:46:46-50.
95 Pagano G, Castello G, Pallardo FV. Sjogren's syndrome-associated oxidative stress and mitochondrial dysfunction: prospects for chemoprevention trials. Free Radic Res 2013;47:71-3.

96 Cordero MD, de Miguel M, Moreno-Fernandez AM. [Mitochondrial dysfunction in fibromyalgia and its implication in the pathogenesis of disease]. Med Clin (Barc) 2011;136:252-6.

97 Sui BD, Xu TQ, Liu JW, et al. Understanding the role of mitochondria in the pathogenesis of chronic pain. Postgrad Med J 2013;89:709-14. 